Article

\title{
Identification of Human \\ UDP-Glucuronosyltransferase 1A4 as the Major Isozyme Responsible for the Glucuronidation of 20(S)-Protopanaxadiol in Human Liver Microsomes
}

\author{
Jia Li ${ }^{1}$, Chunyong He ${ }^{1}$, Lianxiang Fang ${ }^{2}$, Li Yang ${ }^{2,3, *}$ and Zhengtao Wang ${ }^{1,2,3, *}$ \\ 1 Department of Pharmacognosy, China Pharmaceutical University, Nanjing 210038, China; \\ lisa02252@163.com (J.L.); 13764556163@126.com (C.H.) \\ 2 The Ministry Of Education Key Laboratory for Standardization of Chinese Medicines and \\ the SATCM Key Laboratory for New Resources and Quality Evaluation of Chinese Medicines, \\ Institute of Chinese Materia Medica, Shanghai University of Traditional Chinese Medicine, \\ Shanghai 201203, China; lianxiang1205@163.com \\ 3 Institute of Chinese Materia Medica, Shanghai University of Traditional Chinese Medicine, \\ Shanghai 201210, China \\ * Correspondence: yl7@shutcm.edu.cn (L.Y.); ztwang@shutcm.edu.cn (Z.W.); \\ Tel.: +86-21-5132-2506 (L.Y. \& Z.W.); Fax: +86-21-5132-2519 (L.Y. \& Z.W.)
}

Academic Editor: Chang Won Choi

Received: 16 December 2015; Accepted: 26 January 2016; Published: 9 March 2016

\begin{abstract}
S)-protopanaxadiol (PPD), one of the representative aglycones of ginsenosides, has a broad spectrum of pharmacological activities. Although phase I metabolism has been investigated extensively, information regarding phase II metabolism of this compound remains to be elucidated. Here, a glucuronidated metabolite of PPD in human liver microsomes (HLMs) and rat liver microsomes (RLMs) was unambiguously identified as PPD-3-O- $\beta$-D-glucuronide by nuclear magnetic resonance spectroscopy and high resolution mass spectrometry. The chemical inhibition and recombinant human UDP-Glucuronosyltransferase (UGT) isoforms assay showed that the PPD glucuronidation was mainly catalyzed by UGT1A4 in HLM, whereas UGT1A3 showed weak catalytic activity. In conclusion, PPD-3-O- $\beta$-D-glucuronide was first identified as the principal glucuronidation metabolite of PPD in HLMs, which was catalyzed by UGT1A4.
\end{abstract}

Keywords: UGT1A4; liver microsomes; 20(S)-protopanaxadiol; glucuronidation; liquid chromatography-mass spectrometry (LC-MS)

\section{Introduction}

Triterpenoid saponins exist widely in many medicinal plants including ginseng, America ginseng, and notoginseng. Many saponins like ginsenosides might be totally or partially hydrolyzed in gut after oral administration. As a result, the saponins are normally considered as the prodrugs and the aglycones may act as the real pharmacophores exhibiting related biological activities [1]. Therefore, the evaluation of metabolites identification and metabolic pathway of the aglycones would provide a feasible access to these bioactive triterpenoid saponins for further understanding and application.

20(S)-protopanaxadiol (PPD) is one of the representative aglycones of ginsenosides metabolized from numerous dammarane type saponins through stepwise deglycosylation catalyzed by gastric acid or gut organisms. It has been stated that PPD may be the actual executers of ginsenosides [1-4]. PPD itself also has a wide spectrum of pharmacological activities, such as anti-inflammatory [5], antidepressant [6] and anticancer [7-9]. PPD is one of the most potent anticancer agents among 
the ginsenosides proved by the structure-activity relationship study, which can induce Hep G2 cell apoptosis by the endoplasmic reticulum stress pathway [10,11].It is reported that PPD has been regarded as a potential antidepressant by early clinical trials [12].

Previous studies disclosed that oral bioavailability of PPD was determined to be approximate $30 \%$ [13], and it was mainly eliminated via oxidation of the double bond at $\Delta^{(24,25)}$ to yield 24,25-expoides, followed by hydrolysis and rearrangement to form the 24,25-vicinal diol and the 20,24-oxide derivatives [2,14]. Yet the information related to the phase II metabolism of PPD is very limited. Li, et al. [2] reported that direct glucuronidation of PPD only occurred in pooled human liver microsomes and human hepatocytes when it was transformed into 20,24-oxide form. In our previous study, a glucuronidation metabolite was detected in rat urine, bile and plasma [15]. However, the location of the glucuronic acidmoiety and the isozyme(s) catalyzing this reaction remain unknown. Glucuronidation has been increasingly recognized as an elimination and detoxification for xenobiotics and endogenous compounds, which accounts for more than $35 \%$ of all phase II drug metabolism, where the UDP-Glucuronosyltransferases (UGTs) play an important role [16]. Until now, information regarding the glucuronidation of PPD is still limited. Our present study aims at identifying glucuronidation metabolite of PPD and characterizing the UGT isozyme(s) catalyzing the metabolism in human liver microsomes (HLMs) and rat liver microsomes (RLMs), in order to assist the understanding of elimination of PPD as well as PPD-type ginsenosides.

\section{Results}

\subsection{Identification and Structural Elucidation of Metabolite}

A new metabolite designated as PPDG (glucuronidation metabolite of PPD) was eluted at $16.48 \mathrm{~min}$ by ultra-performance liquid chromatography quadrupole time-of-flight tandem mass spectrometry (UPLC-Q/TOF-MS) when PPD was incubated with pooled HLMs or RLMs in the presence of Uridine $5^{\prime}$-diphosphoglucuronic acid trisodium salt (UDPGA) (Figure 1B). The negative ion mode was used for structure identification because it is more sensitive than positive ion mode for PPD and its metabolite [15].

The Q/TOF-MS showed an exact deprotonated ion $[\mathrm{M}-\mathrm{H}]^{-}$at $m / z 635.4158$ (Calcd. 635.4159, elemental composition of $\mathrm{C}_{36} \mathrm{H}_{59} \mathrm{O}_{9}$ ) (Figure 2A), 176.0322 Da higher than that of PPD, suggesting conjugation with glucuronic acid. In the $\mathrm{MS}^{2}$ (product ions) spectrum, the typical neutral loss $(-176.0322 \mathrm{Da}$ ) of a glucuronic acid moiety was observed from $\mathrm{m} / z 635.4158$ to $\mathrm{m} / \mathrm{z}$ 459.3836. The characteristic ion at $\mathrm{m} / z 375.2898$ generated from the loss of $-\mathrm{CH}_{2} \mathrm{CH}_{2} \mathrm{CH}=\mathrm{C}\left(\mathrm{CH}_{3}\right)_{2}$ at $\mathrm{C}-20$ position (Figure 2B).

A

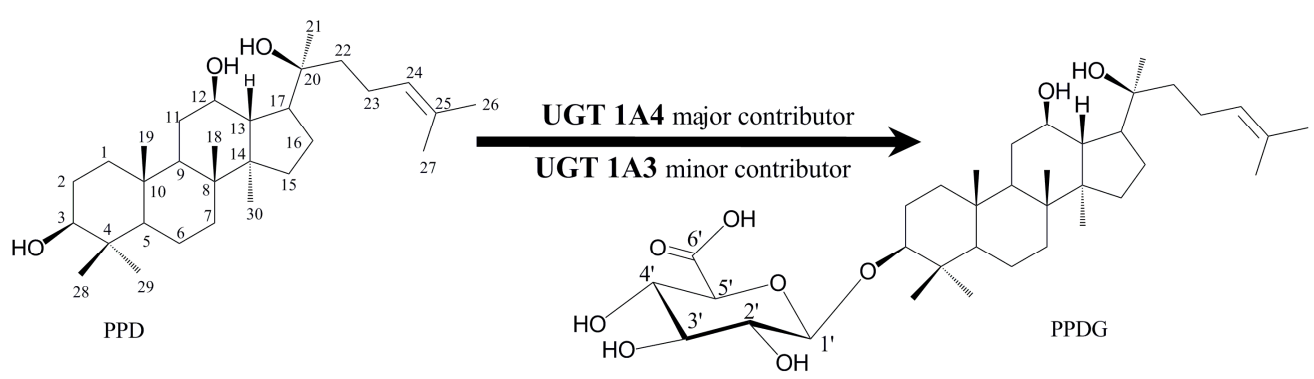

Figure 1. Cont. 


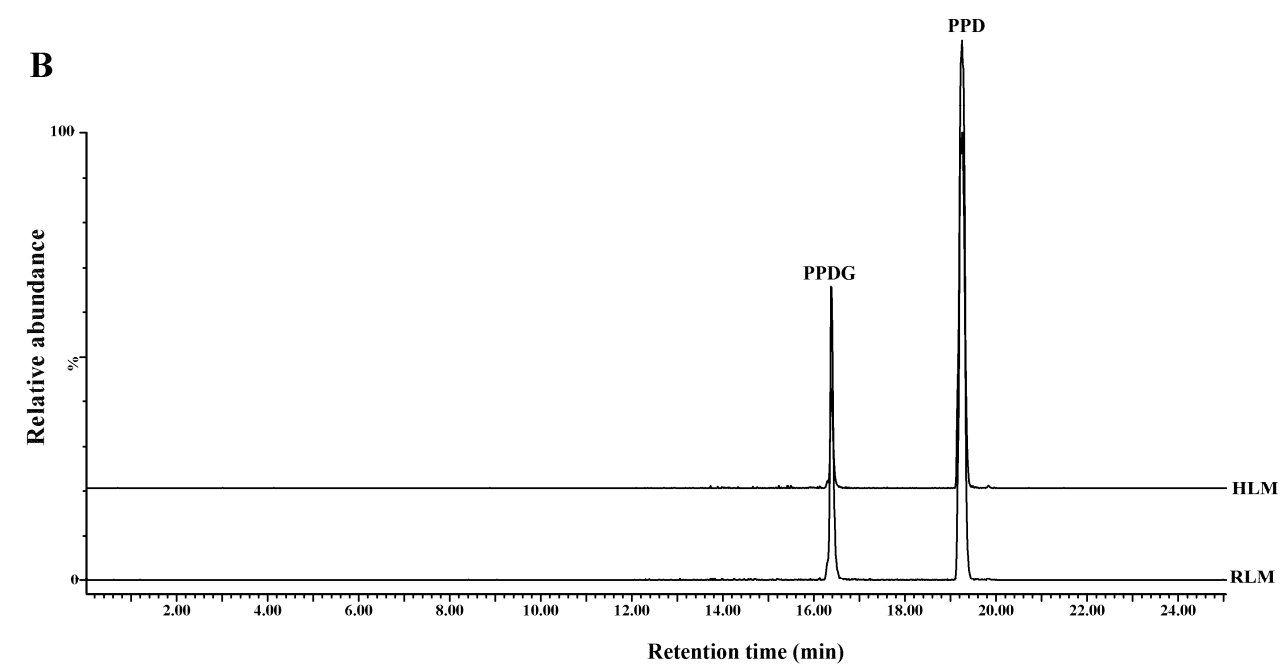

Figure 1. Chemical structures of 20(S)-protopanaxadiol (PPD) and its glucuronidation metabolite catalyzed by human UDP-Glucuronosyltransferase (UGT) 1A3 and UGT 1A4 (A). The contributions of these isozymes in this pathway were identified by recombinant UGT isozymes. Representative combined ultra-performance liquid chromatography quadrupole time-of-flight tandem mass spectrometry (UPLC-Q/TOF-MS) chromatograms of PPD and its glucuronidation metabolite; $0.5 \mathrm{mg} / \mathrm{mL}$ human liver microsomes (HLMs) or rat liver microsomes (RLMs) was incubated with PPD $(100 \mu \mathrm{M})$ at $37^{\circ} \mathrm{C}$ for $1 \mathrm{~h}$ in the presence of Uridine $5^{\prime}$-diphosphoglucuronic acid trisodium salt (UDPGA) (B).

(A)

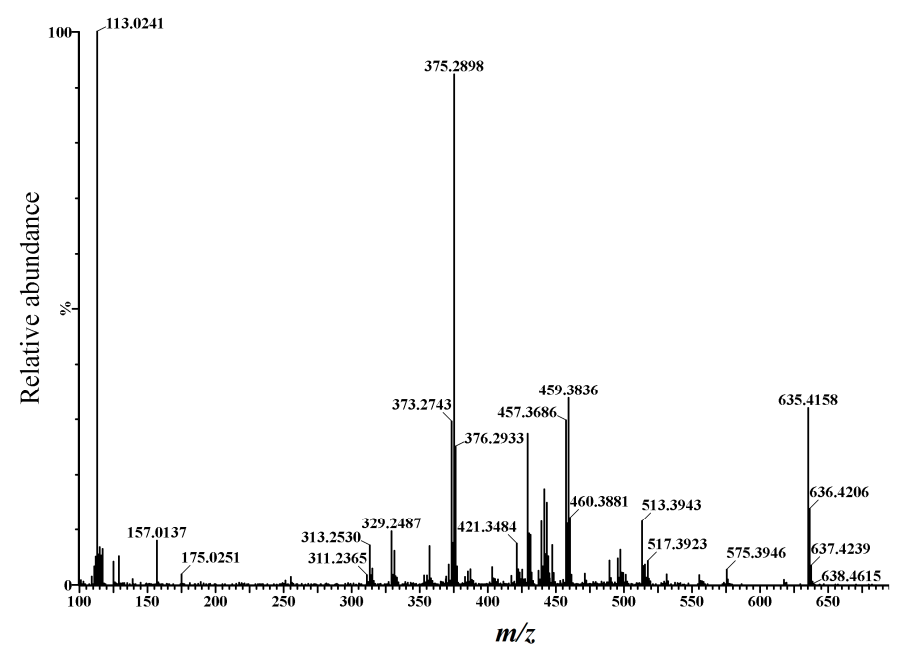

(B)

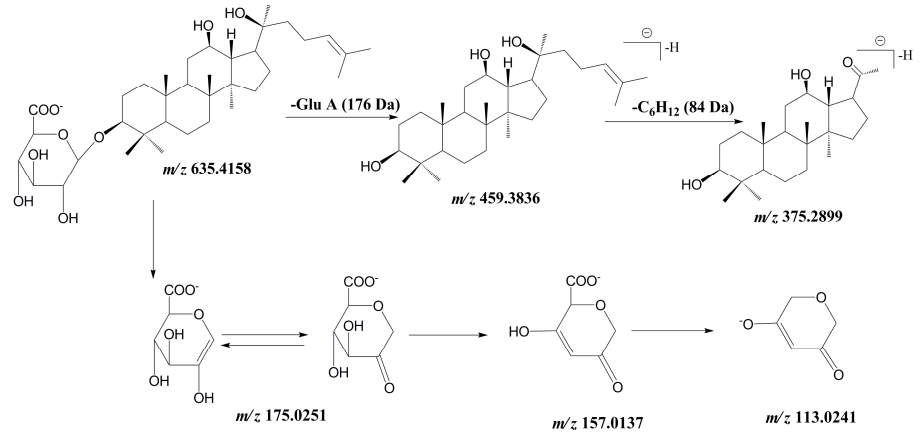

Figure 2. $\mathrm{MS}^{2}$ spectrum of PPDG (A) and its fragmentation pathways $(\mathbf{B})$ in negative ion mode obtained from Q/TOF-MS. The MS² data were obtained with $m / z 635.41\left([\mathrm{M}-\mathrm{H}]^{-}\right)$as the precursor ion. 
To confirm the substitution position of glucuronic acid moiety, standard of this metabolite was further biosynthesized by using pooled RLMs and structurally elucidated by high resolution mass and NMR analyses. In UPLC-Q/TOF-MS analysis, the biosynthesized product showed the identical retention time and $\mathrm{MS}^{2}$ spectrum to those of the microsome-generated product. The ${ }^{1} \mathrm{HNMR}$ and ${ }^{13} \mathrm{CNMR}$ data of PPDG were summarized in Table 1 . Compared with that of PPD, the ${ }^{13} \mathrm{CNMR}$ spectrum of PPDG showed a downfield shift of the C-3 signal $(\Delta \delta+11.7 \mathrm{ppm})$ due to the glycosidation, while the signals at C-12 and C-20 remain unchanged, which indicated the glucuronic acid moiety substitution occurred at C-3 position. The anomeric configuration of the glucuronic acid was confirmed according to its coupling constant of the anomeric proton $(\delta 4.35 \mathrm{ppm}, J=7.8 \mathrm{~Hz})$. Based on the evidence above, this metabolite was identified as PPD-3-O- $\beta$-D-glucuronic acid (Figure 1A).

Table 1. ${ }^{1} \mathrm{H}$ and ${ }^{13} \mathrm{C}$ NMR data of 20(S)-protopanaxadiol (PPD) and its glucuronidation metabolite (PPDG) (600 MHz, methanol- $\left.d_{4}\right)$.

\begin{tabular}{|c|c|c|c|c|}
\hline \multirow{2}{*}{ Position } & \multicolumn{2}{|c|}{${ }^{13} \mathrm{C}$ NMR } & \multicolumn{2}{|c|}{${ }^{1} \mathrm{H}$ NMR } \\
\hline & PPD $^{a}$ & PPDG & PPD $^{a}$ & PPDG \\
\hline 1 & $39.4, \mathrm{t}$ & $39.0, \mathrm{t}$ & - & - \\
\hline 2 & $28.2, \mathrm{t}$ & $29.4, \mathrm{t}$ & - & - \\
\hline 3 & $78.4, \mathrm{~d}$ & $90.1, \mathrm{~d}$ & - & - \\
\hline 4 & $39.5, \mathrm{~s}$ & $38.6, \mathrm{~s}$ & - & - \\
\hline 5 & $56.4, d$ & $56.1, \mathrm{~d}$ & - & - \\
\hline 6 & $18.8, \mathrm{~d}$ & $17.8, \mathrm{~d}$ & - & - \\
\hline 7 & $35.2, \mathrm{t}$ & $34.6, \mathrm{t}$ & - & - \\
\hline 8 & $40.0, d$ & $39.6, d$ & - & - \\
\hline 9 & $50.5, \mathrm{~d}$ & $50.0, \mathrm{~d}$ & - & - \\
\hline 10 & $37.4, \mathrm{~s}$ & $36.6, \mathrm{~s}$ & - & - \\
\hline 11 & $32.1, \mathrm{t}$ & $31.7, \mathrm{t}$ & - & - \\
\hline 12 & $71.0, \mathrm{~d}$ & $71.9, \mathrm{~d}$ & $3.9, \mathrm{~m}$ & $4.1, \mathrm{~m}$ \\
\hline 13 & $48.6, \mathrm{~d}$ & $48.2, \mathrm{~d}$ & - & - \\
\hline 14 & $51.7, \mathrm{~s}$ & $51.2, \mathrm{~s}$ & - & - \\
\hline 15 & $31.4, \mathrm{t}$ & $30.7, \mathrm{t}$ & - & - \\
\hline 16 & $26.8, \mathrm{t}$ & $26.0, \mathrm{t}$ & - & - \\
\hline 17 & $54.8, \mathrm{~d}$ & $53.7, \mathrm{~d}$ & $2.33, J=10.7,7.1^{b}$ & $2.37, \mathrm{dd}, J=15,7.8$ \\
\hline 18 & $15.9, \mathrm{q}$ & $15.4, \mathrm{q}$ & $0.99, \mathrm{~s}$ & $1.06, \mathrm{~s}$ \\
\hline 19 & $16.4, \mathrm{q}$ & $15.7, \mathrm{q}$ & $0.87, \mathrm{~s}$ & $0.87, \mathrm{~s}$ \\
\hline 20 & $72.9, \mathrm{~s}$ & $74.0, \mathrm{~s}$ & - & - \\
\hline 21 & $27.1, \mathrm{q}$ & $27.0, \mathrm{q}$ & $1.41, \mathrm{~s}$ & $1.31, \mathrm{~s}$ \\
\hline 22 & $35.9, \mathrm{t}$ & $35.0, \mathrm{t}$ & - & - \\
\hline 23 & $23.0, \mathrm{t}$ & $24.5, \mathrm{t}$ & $2.28, \mathrm{~s}$ & - \\
\hline 24 & $126.3, \mathrm{~d}$ & $125.9, \mathrm{~d}$ & $5.3, J=7.1$ & $5.1, \mathrm{dd}, J=7.6,1.2$ \\
\hline 25 & $130.7, \mathrm{~s}$ & $130.6, \mathrm{~s}$ & - & - \\
\hline 26 & $25.8, \mathrm{q}$ & $25.9, \mathrm{q}$ & $1.64, \mathrm{~s}$ & $1.70, \mathrm{~s}$ \\
\hline 27 & $17.7, \mathrm{q}$ & $15.7, \mathrm{q}$ & $1.61, \mathrm{~s}$ & $1.64, \mathrm{~s}$ \\
\hline 28 & $28.7, \mathrm{q}$ & $29.1, \mathrm{q}$ & $1.21, \mathrm{~s}$ & $1.16, \mathrm{~s}$ \\
\hline 29 & $16.3, \mathrm{q}$ & $14.8, \mathrm{q}$ & $1.02, \mathrm{~s}$ & $1.03, \mathrm{~s}$ \\
\hline 30 & $17.0, \mathrm{q}$ & $15.4, \mathrm{q}$ & $0.92, \mathrm{~s}$ & $0.94, \mathrm{~s}$ \\
\hline & & & Glucuronic acid & \\
\hline $1^{\prime}$ & - & $106.4, \mathrm{~d}$ & - & $4.35, \mathrm{~d}, J=7.8$ \\
\hline $2^{\prime}$ & - & $76.3, \mathrm{~d}$ & - & - \\
\hline $3^{\prime}$ & - & $77.7, \mathrm{~d}$ & - & - \\
\hline $4^{\prime}$ & - & $73.5, \mathrm{~d}$ & - & - \\
\hline $5^{\prime}$ & - & $75.2, \mathrm{~d}$ & - & - \\
\hline $6^{\prime}$ & - & $175.3, \mathrm{~s}$ & - & - \\
\hline
\end{tabular}

${ }^{\mathrm{a}}$ Reporeted by [17]; ${ }^{\mathrm{b}}$ Coupling constant expressed as Hz. 
2.2. Kinetics of 20(S)-Protopanaxadiol (PPD) Glucuronidation by Pooled Human Liver Microsomes (HLMs) and Rat Liver Microsomes (RLMs)

Apparent enzyme kinetic parameters were evaluated by incubating PPD $(10-500 \mu \mathrm{M})$ with pooled HLMs and RLMs. Sigmoidal kinetic model was best fitted to PPD glucuronidation by HLMs and RLMs (Hill model, Goodness of fit $R^{2}>0.97$ ) with the Hill coefficient of $>1$ (positive cooperation), as evidenced by the Eadie-Hofstee plot (Figure 3). All the kinetic parameters are summarized in Table 2. The maximum formation rate of PPDG in the incubation containing HLMs was of $0.32 \pm 0.01 \mathrm{nmol} / \mathrm{min} / \mathrm{mg}$ protein, whereas the $S_{50}$ (substrate concentration at $0.5 V_{\max }$ (analogous to $K_{\mathrm{m}}$ in the Michaelis-Menten equation)) and $n$ (Hill coefficient (which reflects the degree of sigmoidicity of the velocity versus substrate-concentration relationship)) of Hill component were of $42.80 \pm 0.73 \mu \mathrm{M}$, and $2.12 \pm 0.24$, respectively. When PPD was incubated with RLMs, the maximum formation rate of PPDG was comparable with that in HLMs, whereas the $S_{50}$ value of PPDG in RLMs was approximately $50 \%$ of that in HLMs. The maximum clearance in HLMs and RLMs were calculated to be $3.70 \pm 0.01$, $7.36 \pm 0.27 \mu \mathrm{L} / \mathrm{min} / \mathrm{mg}$ protein, respectively.
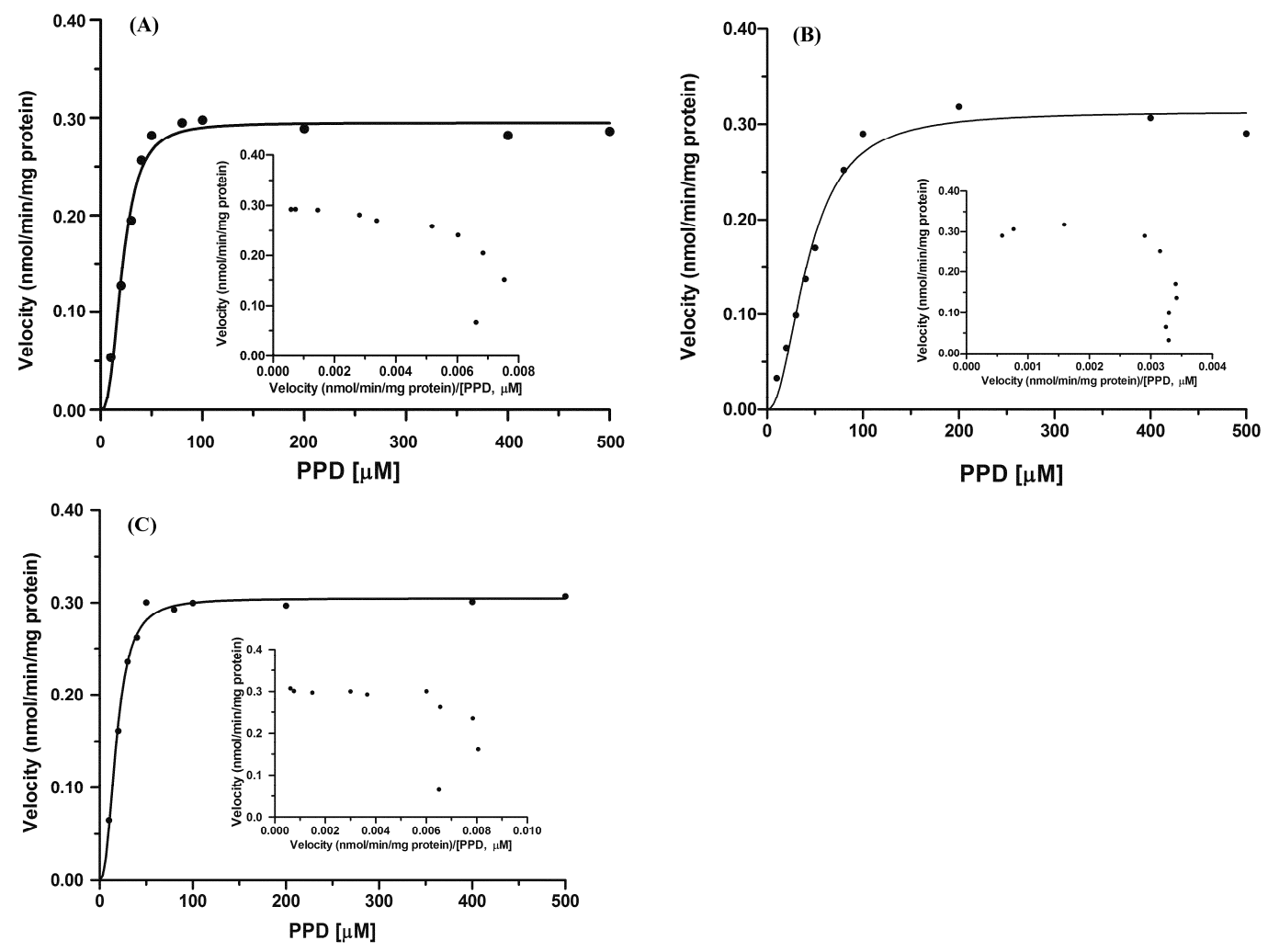

Figure 3. Enzyme kinetics of PPD glucuronidation by rat liver microsomes (RLMs) (A); human liver microsomes (HLMs) (B) and recombinant UGT1A4 (C). PPD (10-500 $\mu \mathrm{M})$ was incubated with HLMs and RLMs (0.5 mg protein/mL) in the presence of UDPGA. An Eadie-Hofstee plot is shown as an inset. Data were obtained from three replicates.

Table 2. Kinetic parameters for the formation of PPDG with pooled human liver microsomes (HLMs), rat liver microsomes (RLMs), and recombinant human UGT1A4 (Enzyme kinetic parameters were determined by Hill kinetics at 10-500 $\mu \mathrm{M}$ PPD. Data were expressed as mean $\pm \mathrm{SD}$ ).

\begin{tabular}{cccccc}
\hline Species & $\boldsymbol{V}_{\max }(\mathbf{n m o l} / \mathbf{m i n} / \mathbf{m g}$ Protein) & $\boldsymbol{S}_{\mathbf{5 0}}(\mu \mathbf{M})$ & $\boldsymbol{n}$ & $C_{L_{\max }}(\mu \mathrm{L} / \mathbf{m i n} / \mathbf{m g}$ Protein) & $\boldsymbol{R}^{\mathbf{2}}$ \\
\hline RLMs & $0.30 \pm 0.01$ & $20.68 \pm 1.39$ & $2.50 \pm 0.48$ & $7.36 \pm 0.27$ & 0.9793 \\
HLMs & $0.32 \pm 0.01$ & $42.80 \pm 0.73$ & $2.12 \pm 0.24$ & $3.70 \pm 0.01$ & 0.9753 \\
UGT1A4 & $0.31 \pm 0.01$ & $18.49 \pm 1.85$ & $2.34 \pm 0.23$ & $8.39 \pm 0.79$ & 0.9894 \\
\hline
\end{tabular}




\subsection{Chemical Inhibition in Pooled HLMs}

Four potent chemical inhibitors including fluconazole, androsterone, estradiol, and hecogenin for UGT isozymes were used to inhibit PPD glucuronidation at concentrations of three levels $(5,50$, and $200 \mu \mathrm{M})$. The result (Figure $4 \mathrm{~A})$ indicated that hecogenin showed concentration-dependent inhibitory effect on PPD glucuronidation by more than $60 \%$ compared with the solvent control at $50 \mu \mathrm{M}$; it is regared as most potent inhibiter on this biotransformation. The $\mathrm{IC}_{50}$ was determined to be $21.32 \pm 4.31 \mu \mathrm{M}$ (Figure 4B). Fluconazole, a selective inhibitor of UGT2B7, showed no inhibition on PPD glucuronidation, estradiol and androsterone showed weak inhibition of PPD glucuronidation. The results above indicated that UGT1A4 played a remarkable role in PPD glucuronidation.
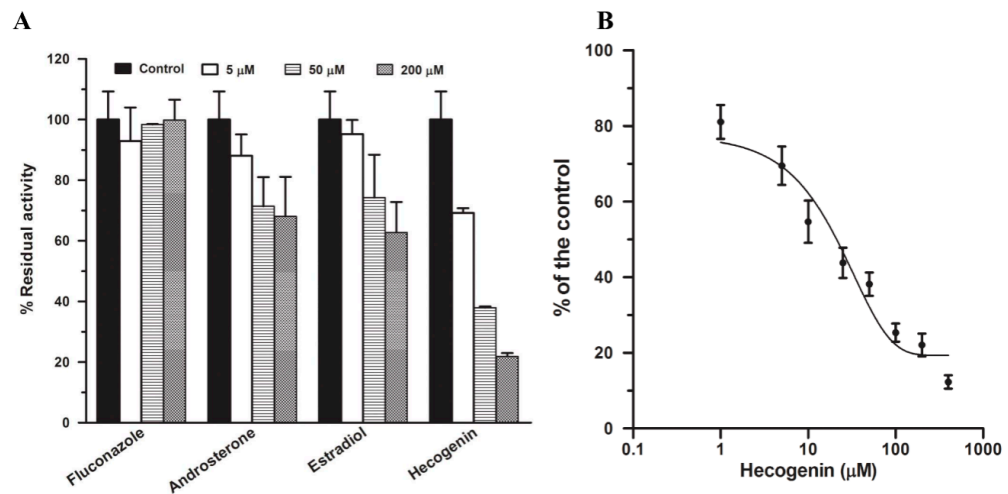

Figure 4. Chemical inhibition of PPD glucuronidation at a PPD concentration of $50 \mu \mathrm{M}$ by four potent inhibitors $(5,50$, and $200 \mu \mathrm{M})$ including fluconazole, hecogenin, estradiol, and androsterone in pooled HLMs $(0.5 \mathrm{mg} / \mathrm{mL})$ incubations. The incubations without inhibitors but the same volume of solvent were set as the control in which the activity of PPD glucuronidation was designated as $100 \%$ (A); Inhibitory effects of hecogenin on PPD glucuronidation. PPD glucuronidation was evaluated in pooled HLMs at the concentration of $50 \mu \mathrm{M}$ in the presence of hecogenin (1-400 $\mu \mathrm{M})(\mathbf{B})$. All data were obtained from three replicates.

\subsection{Assay with Recombinant Human UGTs}

To confirm the results obtained from the chemical inhibition study, PPD glucuronidation was assayed in twelve recombinant UGT isozymes, i.e., UGT1A1, 1A3, 1A4, 1A6, 1A7, 1A8, 1A9,1A10, 2B4, 2B7, 2B15, and 2B17, at $0.25 \mathrm{mg}$ protein/mL (Figure 5). With the PPD concentration of 10, 50 , and $200 \mu \mathrm{M}$, UGT1A4 showed the highest and predominant catalytic activity $(0.06 \pm 0.01$, $0.18 \pm 0.01$, and $0.35 \pm 0.05 \mathrm{nmol} / \mathrm{min} / \mathrm{mg}$ protein, respectively) towards to PPD glucuronidation. With the exception of UGT1A4, UGT1A3 also exhibited slight catalytic activity $(0.02 \pm 0.004$, $0.04 \pm 0.004,0.04 \pm 0.003 \mathrm{nmol} / \mathrm{min} / \mathrm{mg}$ protein at 10,50, and $200 \mu \mathrm{M}$, respectively). Other UGT isozymes tested did not participate in this metabolism.

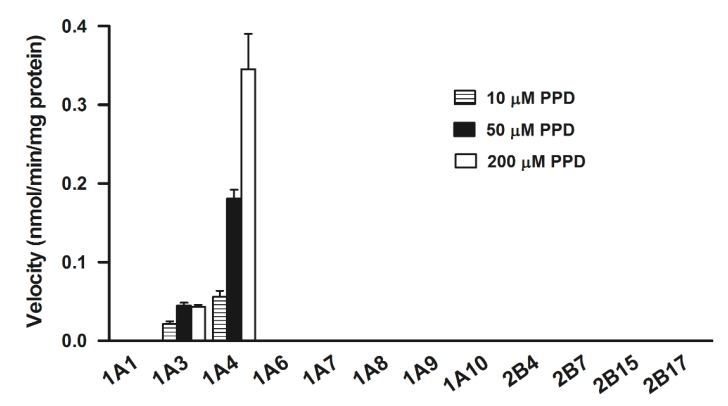

Figure 5. Assays of glucuronidation activities of $\operatorname{PPD}(10,50$, and $200 \mu \mathrm{M})$ catalyzed by different recombinant UGT isozymes $(0.25 \mathrm{mg} / \mathrm{mL})$. Data were obtained from three replicates. 


\subsection{Kinetics of PPD Glucuronidation by Recombinant UGT1A4}

Because UGT1A4 showed the highest PPD glucuronidation activity, kinetic analysis for PPD glucuronidation in recombinant UGT1A4 was conducted using a wide range of concentrations $(10-500 \mu \mathrm{M})$ of PPD. The PPDG formation by UGT1A4, as indicated by the Eadie-Hofstee plot (Figure 3C), exhibited Hill kinetics with the Hill coefficient of $>1$ (positive cooperation). The maximum formation rate was of $0.31 \pm 0.01 \mathrm{nmol} / \mathrm{min} / \mathrm{mg}$ protein, whereas the mean $S_{50}$ was of $18.49 \pm 1.85 \mu \mathrm{M}$, and the maximum clearance in recombinant UGT1A4 was of $8.39 \pm 0.79 \mu \mathrm{L} / \mathrm{min} / \mathrm{mg}$ protein.

\section{Discussion}

Ginsenosides and notoginsenosides were demonstrated to be the main bioactive components of Panax notoginseng and its corresponding preparations, which exhibited pharmacological activity against cardiovascular and cerebrovascular disorders [18-20]. Metabolism and pharmacokinetic study suggested that both PPD (protopanaxadiol) and PPT (protopanaxatriol)-type ginsenosides and notoginsenosides are susceptible to deglycosylation metabolism by gastric acid and/or intestinal bacteria in vivo [21,22]. PPD and PPT have been widely regarded as the final metabolites through the gastrointestinal tract, which may be responsible for the pharmacological activity of ginsenosides and notoginsenosides [1-4]. Currently, PPD is regarded as a potential antidepressant by early clinical trials [12]. However, information regarding the metabolic pathway of PPD after absorption from the digestive tract is very limited. In vitro phase I metabolism indicated that the predominant metabolic pathway of PPD was the oxidation of the double bond at $\Delta^{(24,25)}$ to yield 24,25-expoides, followed by hydrolysis and rearrangement to form the 24,25-vicinal diol derivatives and the 20,24-oxide form. PPD could not undergo direct glucuronidation which only occurred when it was transformed into 20,24-oxide form [2,14]. In our study, a glucuronidation metabolite of PPD (designated as PPDG) was first found and identified in pooled HLMs and RLMs using UPLC-Q/TOF-MS in negative ion mode. The chemical structure of PPDG was elucidated based on its accurate mass, mass spectral fragmentation patterns and NMR data. Our experiment suggested that hydroxyl group at C-3 position was susceptible to glucuronidation.

To identify and characterize the UGT isozymes that involved in PPD glucuronidation, optimization of incubation conditions in terms of linearity of metabolite formation with increased microsomal protein and incubation time were first performed with pooled HLMs. Subsequently, PPD glucuronidation activities in pooled HLMs and RLMs were determined. Then chemical inhibition study and a panel of twelve commercially available recombinant UGTs were used to identify the isozymes involved in PPD glucuronidation and their contributions. Finally, kinetics of PPD glucuronidation was performed in human recombinant UGT1A4. The kinetic profiles in pooled RLMs, HLMs and recombinant UGT1A4 followed the sigmoidal kinetic model (Hill profile), and the Hill coefficient was more than 1 , which indicated that a positive cooperative reaction had occurred.

The selective inhibitors for UGT isozymes have not been identified as extensively as P450 isozymes [23]. To date, only two selective inhibitors have been identified: hecogenin for UGT1A4 [24] and fluconazole for UGT2B7 [25], which were applied in the present study and hecogenin showed the most significant inhibitory effect with the $\mathrm{IC}_{50}$ of $21.32 \pm 4.31 \mu \mathrm{M}$ (Figure 4), suggesting that UGT1A4 was the most important isozyme catalyzing the PPD glucuronidation. Additionally, androsterone and estradiol, as other two potent inhibitors, were also used in this study. It had been demonstrated that androsterone is a potent inhibitor for UGT1A9 and UGT2B7; however, it showed only slight inhibition even at a high concentration, suggesting that UGT1A9 and UGT2B7 were not involved in PPD glucuronidation. It has been reported that androsterone also inhibited UGT1A4 slightly [25], which may be the reason for its slight inhibition of PPD glucuronidation. The phenolic glucuronidation of $\beta$-estradiol at C-3 position is mainly catalyzed by UGT1A1 with atypical kinetics [26,27], whereas the alcoholic glucuronidation of $\beta$-estradiol at C-17 position is mostly catalyzed by UGT2B7 [28]. However, studies indicate UGT1A8, 1A10, and $1 \mathrm{~A} 3$ can also catalyze the glucuronidation of $\beta$-estradiol at C-3 position with significant activities [29], whereas UGT1A3, 1A4, 1A8, 1A10, and 2B7 may also 
take part in the glucuronidation of $\beta$-estradiol at C-17 [29]. In this experiment, estradiol was used as an inhibitor to inhibit PPD glucuronidation. As shown in Figure 4, estradiol showed slight inhibitory effect on PPD glucuronidation, suggesting that UGT1A1, UGT1A3, UGT1A8 and UGT2B7 were not involved in PPD glucuronidation. The slight inhibition may be attributed to the slight inhibition of UGT1A4. All the chemical inhibition study indicated that UGT1A4 was the predominant isozyme catalyzing PPD glucuronidation. The conclusion reached was confirmed by twelve recombinant UGT isozymes analyses. As shown in Figure 5, UGT1A4 showed the highest and predominant catalytic activity. Besides UGT1A4, UGT1A3 also catalyze this metabolism, but the efficiency was rather low. Other isozymes tested did not catalyze this reaction.

Human UGT known to metabolize xenobiotics are the products of two gene families, UGT1 and UGT2, and human hepatic UGT enzymes include UGT 1A1, 1A3, 1A4, 1A6, 1A9, 2B4, 2B7, 2B10, 2B11, 2B15 and 2B17 [16]. Although other isozymes that were not tested in the present study may participate in PPD glucuronidation, it could be concluded that UGT 1A4 was the major isozyme involved in PPD glucuronidation according to chemical inhibition and recombinant isozyme analyses, which was further confirmed by the enzymatic parameters in pooled HLMs that were comparable to those in recombinant UGT $1 \mathrm{~A} 4$.

\section{Experimental Section}

\subsection{Materials}

PPD and hecogenin were obtained from Shanghai PureOne Biotechnology (purity $>98 \%$; Shanghai, China). Ginsenoside Rg1 (GRg1) was provided by the Shanghai R\&D Center for Standardization of Traditional Chinese Medicine (purity $>98 \%$; Shanghai, China). Uridine 5 '-diphosphoglucuronic acid trisodium salt (UDPGA) was purchased from Santa Cruz Biotechnology, Inc. (Santa Cruz, CA, USA). Alamethicin, Tris base, estradiol, fluconazole, and magnesium chloride were purchased from Sigma-Aldrich (St. Louis, MO, USA). Androsterone was obtained from Dr. Ehrenstorfer GmbH (Augsburg, Germany). Pooled human liver microsomes (HLMs, prepared from livers of 20 human donors) and human recombinant UGT 1A1, 1A3, 1A4, 1A6, 1A7, 1A8, 1A9, 1A10, 2B4, 2B7, 2B15, and 2B17 were purchased from BD Gentest ${ }^{\mathrm{TM}}$ (Woburn, MA, USA). Pooled rat liver microsomes (RLMs) were prepared according to the methods described as previous report [30] in our laboratory and protein concentration was determined by Bradford assay. The protein concentration was adjusted to $10 \mathrm{mg} / \mathrm{mL}$ using the Tris- $\mathrm{HCl}(50 \mathrm{mM}, \mathrm{pH} 7.4)$ buffer, and then $1 \mathrm{~mL}$ aliquots were dispensed into labeled tubes and stored at $-80^{\circ} \mathrm{C}$. All other chemicals and reagents were of analytical grade and commercially available. Experiment related to animals was performed on the basis of the National Institute of Health Guidelines on the principles of animal care (2004) and approved by the Institutional Animal Care and Use Committee, Shanghai University of Traditional Chinese Medicine (Shanghai, China; 1 November 2010).

\subsection{Glucuronidation of PPD with the Pooled HLMs and RLMs}

In vitro PPD glucuronidation was in an incubation volume of $100 \mu \mathrm{L}$. Incubation conditions were initially optimized for the linear product formation with respect to protein concentration $(0.2-1 \mathrm{mg} / \mathrm{mL})$, and incubation time (15-120 min). The stock solution of PPD was prepared in dimethyl sulfoxide (DMSO), and the final concentration of DMSO in the incubations was $1 \%(v / v)$. Briefly, the incubation mixture contained HLMs or RLMs (0.5 mg of protein/mL), UDPGA (2 mM), $\mathrm{MgCl}_{2}(4 \mathrm{mM})$, alamethicin $(25 \mu \mathrm{g} / \mathrm{mg}$ protein), PPD $(10-500 \mu \mathrm{M})$, and Tris- $\mathrm{HCl}$ buffer $(50 \mathrm{mM}$, $\mathrm{pH}$ 7.4). The mixture was incubated for $5 \mathrm{~min}$ at $37^{\circ} \mathrm{C}$, and then the reaction was started by the addition of UDPGA and incubated in a shaking water bath for $60 \mathrm{~min}$. The reaction was terminated by addition of $200 \mu \mathrm{L}$ of ice-cold acetonitrile containing GRg1 (internal standard (IS), $0.5 \mu \mathrm{g} / \mathrm{mL}$ ) and then centrifuged at $15,000 \times g$ for $10 \mathrm{~min}$ at $4{ }^{\circ} \mathrm{C}$. The supernatant was evaporated to dryness under nitrogen gas at room temperature, and the residue was reconstituted with $200 \mu \mathrm{L}$ of $20 \%$ acetonitrile. 
After centrifugation at $15,000 \times g$ for $10 \mathrm{~min}$ at $4{ }^{\circ} \mathrm{C}, 5 \mu \mathrm{L}$ of the supernatants was subjected to analysis. Incubations without PPD and UDPGA were served as blank and negative controls, respectively.

\subsection{Identification of the Metabolite by UPLC-Q/TOF-MS}

Identification of metabolite was carried out on ultra-performance liquid chromatography quadrupole time-of-flight tandem mass spectrometry (UPLC-Q/TOF-MS) (Waters Corporation, Milford, MA, USA) as described in detail previously [15,31]. The peak at RT (retention time) $19.30 \mathrm{~min}$ was detected as PPD by Q/TOF-MS analysis (Figure 1B).

\subsection{Preparation and Structural Elucidation of Metabolite}

The glucuronidation metabolite of PPD (PPDG) was prepared by biotransformation and purified by preparative HPLC system (Waters Corporation, Milford, MA, USA) for structural elucidation and quantitative analysis. Enzymatic biotransformation of the target metabolite was conducted using pooled RLMs. In brief, $500 \mu \mathrm{M}$ PPD was incubated with pooled RLMs (1 mg of protein $/ \mathrm{mL}$ ), $50 \mathrm{mM}$ Tris- $\mathrm{HCl}$ (pH 7.4), $4 \mathrm{mM} \mathrm{MgCl}$, Brij 58 (0.5 mg/mg protein), and $2 \mathrm{mM}$ UDPGA in $100 \mathrm{~mL}$ final incubations for $2 \mathrm{~h}$ at $37^{\circ} \mathrm{C}$. The stock solution of PPD $(50 \mathrm{mM})$ was prepared in DMSO. The concentration of organic solvent in the final incubation was $1 \%$. The reaction was terminated by addition of $200 \mathrm{~mL}$ of ice-cold acetonitrile. After removal of protein by centrifugation at $15,000 \times g$ for $10 \mathrm{~min}$ at $4{ }^{\circ} \mathrm{C}$, the supernatant was evaporated to dryness in vacuo.

The residue was dissolved in $10 \mathrm{~mL}$ of $80 \%$ methanol and then subjected to a Waters LC-MS controlled preparative HPLC Auto Purification System (Milford, MA, USA). The system was comprised of a 2767 Sample Manager, a 2545 binary high-pressure LC Pump, a column/fluidic organizer (SFO), a 515 make up pump, a 2489 UV detector, a ZQ single-quadrupole mass spectrometer (Waters Corporation) equipped with a Z-spray electrospray source. The SFO contains an accurate splitter $(1: 10,000)$. The complete system was controlled by MassLynx ${ }^{\mathrm{TM}}$ software version 4.1 (Waters Corporation). The mobile phase consisted of acetonitrile and water with a linear gradient from initially $10 \%$ to $90 \%$ acetonitrile over $10 \mathrm{~min}$. The flow rate was set at $15 \mathrm{~mL} / \mathrm{min}$. The target metabolite was eluted at $6.5 \mathrm{~min}$, and lyophilized to get a white powder.

The structure of metabolite was identified by ${ }^{1} \mathrm{HNMR}$ and ${ }^{13} \mathrm{CNMR}$ spectroscopies. All the experiments were recorded on a Bruker AV 600 NMR spectrometer (Bruker, Newark, Germany). The purified metabolite was stored at $-20^{\circ} \mathrm{C}$ before dissolving in methanol- $d_{4}$ for NMR analysis. Chemical shifts were given on a $\delta$ scale and referenced to Tetramethylsilane (TMS) at $0 \mathrm{ppm}$ for ${ }^{1} \mathrm{H} \mathrm{NMR}$ $(600 \mathrm{MHz})$ and ${ }^{13} \mathrm{C}$ NMR $(150 \mathrm{MHz})$.

\subsection{Quantitative Analysis by LC-MS/MS}

Quantitative analysis was conducted on an Agilent 1290 infinity system (Agilent Technologies, Santa Clara, CA, USA) coupled with an MS/MS detector (6410 B Triple Quad LC/MS; Agilent Technologies) in negative ion mode. The chromatographic separations were performed on an ACQUITY UPLC HSS T 3 column $(100 \mathrm{~mm} \times 2.1 \mathrm{~mm}$ i. d. (inner diameter), $1.8 \mu \mathrm{m})$ thermostated at $45^{\circ} \mathrm{C}$. The mobile phase consisted of $\mathrm{A}(5 \mathrm{mM}$ ammonium acetate in water) and $\mathrm{B}$ (acetonitrile) at a flow rate of $0.4 \mathrm{~mL} / \mathrm{min}$. The gradient elution program was set as follow: $0-2 \mathrm{~min} 20 \%-50 \% \mathrm{~B}, 2-4 \mathrm{~min}$ $50 \%-60 \% \mathrm{~B}, 4-5 \mathrm{~min} 60 \% \mathrm{~B}$ and finally, reconditioning the column with $20 \% \mathrm{~B}$ for $1 \mathrm{~min}$. Optimized ion source parameters were set as follows: gas temperature $325^{\circ} \mathrm{C}$; gas flow $10 \mathrm{~L} / \mathrm{min}$; nebulizer $35 \mathrm{psi}$; capillary voltage $3500 \mathrm{~V}$; fragmentor voltage $265 \mathrm{~V}$ for PPDG and $215 \mathrm{~V}$ for IS; cell accelerator voltage $0 \mathrm{~V}$; dwell time $200 \mathrm{~ms}$. Data were acquired in multiple reaction monitor (MRM) mode for the following transitions: $m / z 635.4$ to $m / z 375.1$ for PPDG and $m / z 799.5$ to $m / z 637.6$ for IS, with the collision energy being set at 50 and $26 \mathrm{~V}$, respectively. 


\subsection{Chemical Inhibition in Pooled HLMs}

Fluconazole [24,25], androsterone [25,32], estradiol [26,33], and hecogenin [25,34] were used to inhibit their potential inhibitory effects on PPD glucuronidation in pooled HLMs. A set of concentrations $(5,50$, and $200 \mu \mathrm{M})$ was used for all four inhibitors. The concentration of PPD was set at $50 \mu \mathrm{M}$. The reaction time and protein concentration were set at $60 \mathrm{~min}$ and $0.5 \mathrm{mg} / \mathrm{mL}$, respectively. All other incubation conditions were the same as those for Glucuronidation of PPD in pooled HLMs described above. The concentration of organic solvent was $1 \%$ of the total incubation volume. An experimental group without inhibitors but with solvents was used as the control, in which the activities of PPD glucuronidation were designated as 100\%. The activities of PPD glucuronidation in the inhibited samples were compared with the control to evaluate the remaining enzyme activity. For determination of $\mathrm{IC}_{50}$ (representing the concentration that inhibits $50 \%$ of the control activity) of hecogenin, experiments with a set of concentrations of hecogenin $(1-400 \mu \mathrm{M})$ in pooled HLMs were conducted.

\subsection{Incubation with Recombinant Human UGTs}

PPD glucuronidation was measured in reaction mixtures containing human recombinant UGT1A1, 1A3, 1A4, 1A6, 1A7, 1A8, 1A9, 1A10, 2B4, 2B7, 2B15, and 2B17. Three substrate concentrations $(10,50$, and $200 \mu \mathrm{M})$ were used in this study. The incubation conditions were the same as those for Glucuronidation of PPD in pooled HLMs described above except for the protein concentration $(0.25 \mathrm{mg} / \mathrm{mL})$.

\subsection{Kinetics of PPD Glucuronidation in Recombinant UGT $1 A 4$}

A kinetic study for PPD glucuronidation by human recombinant UGT 1A4 was also conducted by incubating PPD with human recombinant UGT 1A4 at protein concentration of $0.25 \mathrm{mg} / \mathrm{mL} \mathrm{for} 1 \mathrm{~h}$. All other incubation conditions were identical to those of glucuronidation of PPD in pooled HLMs.

\subsection{Enzyme Kinetic Data Analysis}

All results were obtained from three replicates in different microsomal incubations, and all the data were expressed as mean \pm SD. To estimate the kinetic parameters, data were transformed and Eadie-Hofstee curves were plotted, which help to identify kinetic models. Then kinetic parameters were obtained by fitting velocity data to the sigmoidal kinetic (Hill) model (Equation (1)) using Graph Pad Prism 5.0 software (San Diego, CA, USA). In addition, $C L_{\max }$ was calculated according to the Equation (2).

$$
\begin{gathered}
V=\frac{V_{\max } \times[S]^{n}}{S_{50}^{n}+[S]^{n}} \\
C L_{\max }=\frac{V_{\max }}{S_{50}} \times \frac{n-1}{n(n-1)^{1 / n}}
\end{gathered}
$$

$V$ is reaction velocity, $V_{\max }$ is the maximum reaction velocity, [S] is the substrate concentration, $S_{50}$ is the substrate concentration at $0.5 V_{\max }$ (analogous to $\mathrm{Km}$ in the Michaelis-Menten equation), $n$ is the Hill coefficient (which reflects the degree of sigmoidicity of the velocity versus substrate-concentration relationship), $C L_{\max }$ is the maximum clearance, which provides an estimate of the highest clearance attained.

\section{Conclusions}

In our present study, the glucuronidation of PPD in pooled RLMs and HLMs was first investigated and the metabolite was identified as PPD-3-O- $\beta$-D-glucuronide by comparison with a biosynthesized product of which the structure was identified via UPLC-Q/TOF-MS and NMR analyses. The major 
isozyme involved in PPD glucuronidation was UGT1A4. In addition, UGT1A3 also showed a very slight effect. More studies on the biological activities of this glucuronidation metabolite are needed.

Acknowledgments: This work is financially supported by the Natural Science Foundations of China (81573581 and 81530096) and the Program for New Century Excellent Talents in University (NCET-12-1056). We thank Liu Qing at Shanghai University of Traditional Chinese Medicine, for his kind assistance on the chemical structure elucidation of PPD glucuronide.

Author Contributions: Conceived and designed: Jia Li, Li Yang, Zhengtao Wang; Conducted experiments: Jia Li, Chunyong He; Contributed new reagents or analytic tools: Lianxiang Fang; Performed data analysis: Jia Li, Chunyong He; Wrote the manuscript: Jia Li.

Conflicts of Interest: The authors declare no conflict of interest.

\section{References}

1. Hao, H.; Lai, L.; Zheng, C.; Wang, Q.; Yu, G.; Zhou, X.; Wu, L.; Gong, P.; Wang, G. Microsomal cytochrome p450-mediated metabolism of protopanaxatriol ginsenosides: Metabolite profile, reaction phenotyping, and structure-metabolism relationship. Drug Metab. Dispos. 2010, 38, 1731-1739. [CrossRef] [PubMed]

2. Li, L.; Chen, X.; Li, D.; Zhong, D. Identification of 20(S)-protopanaxadiol metabolites in human liver microsomes and human hepatocytes. Drug Metab. Dispos. 2011, 39, 472-483. [CrossRef] [PubMed]

3. Sun, J.; Wang, G.; Haitang, X.; Hao, L.; Guoyu, P.; Tucker, I. Simultaneous rapid quantification of ginsenoside $\mathrm{Rg}_{1}$ and its secondary glycoside $\mathrm{Rh}_{1}$ and aglycone protopanaxatriol in rat plasma by liquid chromatography-mass spectrometry after solid-phase extraction. J. Pharma. Biomed. Anal. 2005, 38, 126-132. [CrossRef] [PubMed]

4. Wang, C.Z.; Kim, K.E.; Du, G.J.; Qi, L.W.; Wen, X.D.; Li, P.; Bauer, B.A.; Bissonnette, M.B.; Musch, M.W.; Chang, E.B. Ultra-performance liquid chromatography and time-of-flight mass spectrometry analysis of ginsenoside metabolites in human plasma. Am. J. Chin. Med. 2011, 39, 1161-1171. [CrossRef] [PubMed]

5. Lee, W.M.; Kim, S.D.; Kim, K.S.; Song, Y.B.; Kwak, Y.S.; Cho, J.Y.; Park, H.J.; Oh, J.W.; Rhee, M.H. Protopanaxadiol modulates LPS-induced inflammatory activity in murinemacrophage RAW264.7 cells. J. Ginseng Res. 2006, 30, 181-187.

6. Hui, Y.; Yang, Z.; Yang, Z.; Ge, Q. Inventors; CN-knowhow intellectual property agent limited, assignee. Antidepressant Compos. Contain. 2007, 20.

7. Li, G.; Wang, Z.; Sun, Y.; Liu, K.; Wang, Z. Ginsenoside 20(S)-protopanaxadiol inhibits the proliferation and invasion of human fibrosarcoma HT1080 cells. Basic Clin. Pharmacol. 2006, 98, 588-592. [CrossRef] [PubMed]

8. Liu, G.Y.; Bu, X.; Yan, H.; Jia, W.W.G. 20S-protopanaxadiol-induced programmed cell death in glioma cells through caspase-dependent and-independent pathways. J. Nat. Prod. 2007, 70, 259-264. [CrossRef] [PubMed]

9. Zhang, R.; Xu, H.; Yu, X.; Qu, S.; Chen, M.; Sui, D. Effect of 20(S)-protopanaxadiol on SMMC-7721 human liver cancer in vivo and in vitro. Chin. Pharmacol. Bull. 2008, 24, 1504-1508.

10. Qi, L.W.; Wang, C.Z.; Yuan, C.S. American ginseng: Potential structure-function relationship in cancer chemoprevention. Biochem. Pharmacol. 2010, 80, 947-954. [CrossRef] [PubMed]

11. Zhu, G.Y.; Li, Y.W.; Tse, A.K.W.; Hau, D.K.P.; Leung, C.H.; Yu, Z.L.; Fong, W.F. 20(S)-protopanaxadiol, a metabolite of ginsenosides, induced cell apoptosis through endoplasmic reticulum stress in human hepatocarcinoma HepG2 cells. Eur. J. Pharmacol. 2011, 668, 88-98. [CrossRef] [PubMed]

12. Xu, C.; Teng, J.; Chen, W.; Ge, Q.; Yang, Z.; Yu, C.; Yang, Z.; Jia, W. 20(S)-protopanaxadiol, an active ginseng metabolite, exhibits strong antidepressant-like effects in animal tests. Prog. Neuro-Psychopharmacol. Biol. Psychiatry 2010, 34, 1402-1411. [CrossRef] [PubMed]

13. Bao, Y.; Wang, Q.; Tang, P. Lithium adduct as precursor ion for sensitive and rapid quantification of 20(S)-protopanaxadiol in rat plasma by liquid chromatography/quadrupole linear ion trap mass spectrometry and application to rat pharmacokinetic study. J. Mass Spectrom. 2013, 48, 399-405. [CrossRef] [PubMed]

14. Jin, X.; Li, S.L.; Zhang, Z.H.; Zhu, F.X.; Sun, E.; Wei, Y.J.; Jia, X.B. Characterization of metabolites of 20(S)-protopanaxadiol in rats using ultra-performance liquid chromatography/quadrupole-time-of-flight mass spectrometry. J. Chromatogr. B 2013, 933, 59-66. [CrossRef] [PubMed] 
15. He, C.; Li, J.; Wang, R.; Li, Z.; Annie Bligh, S.; Yang, L.; Wang, Z. Metabolic profiles of 20(S)-protopanaxadiol in rats after oral administration using ultra-performance liquid chromatography/quadrupole time-of-flight tandem mass spectrometry. Rapid Commun. Mass Spectrom. 2014, 28, 595-604. [CrossRef] [PubMed]

16. Kiang, T.K.; Ensom, M.H.; Chang, T.K.H. UDP-glucuronosyltransferases and clinical drug-drug interactions. Pharmacol. Ther. 2005, 106, 97-132. [CrossRef] [PubMed]

17. Ma, S.G.; Jiang, Y.T.; Song, S.J.; Wang, Z.H.; Bai, J.; Xu, S.X.; Liu, K. Alkaline-degradation products of ginsenosides from leaves and stems of Panax quinquefolium. Acta Pharm. Sin. 2005, 40, 924-930.

18. Cai, B.X.; Li, X.Y.; Chen, J.H.; Tang, Y.B.; Wang, G.L.; Zhou, J.G.; Qui, Q.Y.; Guan, Y.Y. Ginsenoside-Rd, a new voltage-independent $\mathrm{Ca}^{2+}$ entry blocker, reverses basilar hypertrophic remodeling in stroke-prone renovascular hypertensive rats. Eur. J. Pharmacol. 2009, 606, 142-149. [CrossRef] [PubMed]

19. Liu, H.F.; Yang, J.L.; Du, F.F.; Gao, X.M.; Ma, X.T.; Huang, Y.H.; Xu, F.; Niu, W.; Wang, F.Q.; Mao, Y.; et al . Absorption and disposition of ginsenosides afteroral administration of Panax notoginseng extract to rats. Drug Metab. Dispos. 2009, 37, 2290-2298. [CrossRef] [PubMed]

20. Chen, S.; Liu, J.; Liu, X.; Fu, Y.; Zhang, M.; Lin, Q.; Zhu, J.; Mai, L.; Shan, Z.; Yu, X.; et al. Panax notoginseng saponins inhibit ischemia-induced apoptosis by activating PI3K/Akt pathway in cardiomyocytes. J. Ethnopharmacol. 2011, 137, 263-270. [CrossRef] [PubMed]

21. Kong, H.; Wang, M.; Venema, K.; Maathuis, A.; Heijden, R.; Greef, J.; Xu, G.W.; Hankemeier, T. Bioconversion of red ginseng saponins in the gastro-intestinal tract in vitro model studied by high-performance liquid chromatography-high resolution Fourier transform ion cyclotron resonance mass spectrometry. J. Chromatogr. A 2009, 1216, 2195-2203. [CrossRef] [PubMed]

22. Wang, H.Y.; Hua, H.Y.; Liu, X.Y.; Liu, J.H.; Yu, B.Y. In vitro biotransformation of red ginseng extract by human intestinal microflora: Metabolites identification and metabolic profile elucidation using LC-Q-TOF/MS. J. Pharm. Biomed. 2014, 98, 296-306. [CrossRef] [PubMed]

23. Bjornsson, T.D.; Callaghan, J.T.; Einolf, H.J.; Fischer, V.; Gan, L.; Grimm, S.; Kao, J.; King, S.P.; Miwa, G. The conduct of in vitro and in vivo drug-drug interaction studies: A Pharmaceutical Research and Manufacturers of America (PhRMA) perspective. Drug Metab. Dispos. 2003, 31, 815-832. [CrossRef] [PubMed]

24. Liu, H.X.; He, Y.Q.; Hu, Y.; Liu, Y.; Zhang, J.W.; Li, W.; Wang, Z.T.; Yang, L. Determination of UDP-glucuronosyltransferase UGT2B7 activity in human liver microsomes by ultra-performance liquid chromatography with MS detection. J. Chromatogr. B 2008, 870, 84-90. [CrossRef] [PubMed]

25. Uchaipichat, V.; Mackenzie, P.I.; Elliot, D.J.; Miners, J.O. Selectivity of substrate (trifluoperazine) and inhibitor (amitriptyline, androsterone, canrenoic acid, hecogenin, phenylbutazone, quinidine, quinine, and sulfinpyrazone) “probes” for human UDP-glucuronosyltransferases. Drug Metab. Dispos. 2006, 34, 449-456. [PubMed]

26. Soars, M.G.; Ring, B.J.; Wrighton, S.A. The effect of incubation conditions on the enzyme kinetics of UDP-glucuronosyltransferases. Drug Metab. Dispos. 2003, 31, 762-767. [CrossRef] [PubMed]

27. Senafi, S.B.; Clarke, D.J.; Burchell, B. Investigation of the substrate specificity of a cloned expressed human bilirubin UDP-glucuronosyltransferase: UDP-sugar specificity and involvementin steroid and xenobiotic glucuronidation. Biochem. J. 1994, 303, 233-240. [CrossRef] [PubMed]

28. Gall, W.E.; Zawada, G.; Mojarrabi, B.; Tephly, T.R.; Green, M.D.; Coffman, B.L.; Mackenzie, P.I.; Radominska, P.A. Differential glucuronidation of bile acids androgens and estrogens by human UGT1A3 and 2B7. J. Steroid Biochem. Mol. Biol. 1999, 70, 101-108. [CrossRef]

29. Soars, M.G.; Petullo, D.M.; Eckstein, J.A.; Kasper, S.C.; Wrighton, S.A. An assessment of UDP-glucuronosyltransferase induction using primary human hepatocytes. Drug Metab. Dispos. 2004, 32, 140-148. [CrossRef] [PubMed]

30. Zhang, J.W.; Liu, Y.; Zhao, J.Y.; Wang, L.M.; Ge, G.B.; Gao, Y.; Li, W.; Liu, H.T.; Liu, H.X.; Zhang, Y.Y. Metabolic profiling and cytochrome 450 reaction phenotyping of medroxyprogesterone acetate. Drug Metab. Dispos. 2008, 36, 2292-2298. [CrossRef] [PubMed]

31. He, C.; Zhou, D.; Li, J.; Han, H.; Ji, G.; Yang, L.; Wang, Z. Identification of 20(S)-protopanaxatriol metabolites in rats by ultra-performance liquid chromatography coupled with electrospray ionization quadrupole time-of-flight tandem mass spectrometry and nuclear magnetic resonance spectroscopy. J. Pharm. Biomed. 2014, 88, 497-508. [CrossRef] [PubMed] 
32. Liu, H.X.; Liu, Y.; Zhang, J.W.; Li, W.; Liu, H.T.; Yang, L. UDP-glucuronosyltransferase 1A6 is the major isozyme responsible for protocatechuic aldehyde glucuronidation in human liver microsomes. Drug Metab. Dispos. 2008, 36, 1562-1569. [CrossRef] [PubMed]

33. Wen, Z.; Tallman, M.N.; Ali, S.Y.; Smith, P.C. UDP-glucuronosyltransferase 1A1 is the principal enzyme responsible for etoposide glucuronidation in human liver and intestinal microsomes: Structural characterization of phenolic and alcoholic glucuronides of etoposide and estimation of enzyme kinetics. Drug Metab. Dispos. 2007, 35, 371-380. [CrossRef] [PubMed]

34. He, Y.Q.; Liu, Y.; Zhang, B.F.; Liu, H.X.; Lu, Y.L.; Yang, L.; Xiong, A.Z.; Xu, L.L.; Wang, C.H.; Yang, L. Identification of the UDP-glucuronosyltransferase isozyme involved in senecionine glucuronidation in human liver microsomes. Drug Metab. Dispos. 2010, 38, 626-634. [CrossRef] [PubMed]

(C) 2016 by the authors; licensee MDPI, Basel, Switzerland. This article is an open access article distributed under the terms and conditions of the Creative Commons by Attribution (CC-BY) license (http://creativecommons.org/licenses/by/4.0/). 